\title{
Defect Feature Extraction of Marine Protective Coatings by
}

\section{Terahertz Pulsed Imaging}

\author{
Wanli Tu ${ }^{1,3}$, Shuncong Zhong ${ }^{2,3}$ *, Atilla Incecik ${ }^{4}$, Xibin Fu ${ }^{5}$ \\ ${ }^{I}$ Marine Engineering Institute, Jimei University, Xiamen, 361021, P.R. China \\ ${ }^{2}$ School of Mechatronic Engineering and Automation, Shanghai University, Shanghai 200072, P. R. China \\ ${ }^{3}$ Laboratory of Optics, Terahertz and Non-destructive Testing, School of Mechanical Engineering and Automation, \\ Fuzhou University, Fuzhou, 350108, P.R. China \\ ${ }^{4}$ Department of Naval Architecture, Ocean and Marine Engineering, University of Strathclyde, Glasgow G4 OLZ, United Kingdom \\ ${ }^{5}$ Xiamen Special Equipment Inspection Institute, Xiamen 361000, P. R. China \\ *Corresponding author: zhongshuncong@hotmail.com
}

\begin{abstract}
Feature extraction of marine protective coatings using Hilbert transform (HT) and wavelet transform modulus maximum (WTMM) on terahertz pulsed imaging (TPI) of the coatings was proposed. For TPI-based marine protective coating detection, it is difficult to locate exactly the reflected echoes form internal structure of coating system by the faint feature in the time domain due to the interference of background noise. However, those faint features were usually caused by the interface between two different medium layers whose refractive indices are very similar or caused by micro defects beneath the coating. The proposed algorithm was validated by simulated and experimental TPI waveform obtained from marine paint samples with or without defects. To extract the structure feature more clearly and intuitively, Hilbert transform procedure was carried out on detected terahertz signal to get Hilbert envelope for further processing. Subsequently, the modulus maximum of the stationary wavelet transform approximation coefficients were employed as the criteria for feature extraction of internal interfaces and defect features, according to the relationship between WTMM and signal singularity. The results demonstrated that the combination of HT and WTMM algorithms could be used to exactly extract the structure feature and to evaluate the position of defects beneath coatings.
\end{abstract}


Keywords: Marine Protective Coatings, Terahertz Pulsed Imaging, Stationary Wavelet Transform, Modulus Maximum, Hilbert transform

\section{Introduction}

As a non-invasive coherent optical imaging modality and with the ability of preserving the timegated phase information, terahertz pulsed imaging (TPI) can be applied to characterize the internal structures of a sample quantitatively and non-destructively. It could explore the dielectric and optical properties of a range of materials including polar/nonpolar liquids, gases, semiconductors and dielectrics. It provides the capability for standoff inspection of protective coatings that are otherwise opaque at visible and infrared wavelengths [1]. Thus it has great potential in protective coating testing in industry. The utility of time-domain terahertz technology for automobile paint thickness measurements has previously been demonstrated in the laboratory by T. Yasui et al. [2-3]. T. Fukuchi and coworkers determined the refractive index and thickness of the topcoat of a thermal barrier coating from the reflected waveforms of terahertz waves [4]. David J. Cook and coworkers discussed the applicability of terahertz non-destructive testing for marine protective coatings and took a laboratory investigation to measure the dry film thickness of organic coatings. They were developing a standoff sensor based on THz pulsed imaging system in reflected mode for the real-time thickness measurement of wet (uncured) marine paints for the purpose of providing feedback to an automated system for painting ships in drydock. However, the studies were not cover the embedded defects detection and feature extraction for multilayered medium [5-6].

During the corrosive deterioration process of marine protective coating, the chemical and physical characteristics changes in the coating system and thus forms various defects, such as bubbling, rust, cracking, shedding etc. Most sever corrosion degradation which may lead to the replacement of a complete ship panel (deck, side, bottom, etc.) are developed by micro defects [7-8]. It has been reported 
that the corrosion rate is predicted to be higher when the coating defects are smaller [9]. It is of practical importance to detect possible coating defects and evaluate coating performance for monitoring the degradation of paint layers and providing timely and effective maintenance to avoid serious consequences due to coating failure [10]. For example, paint-off (detachment or paint bulge) defect, one of the most commonest coating defects, is the separation of the paint layer from the substrate or other paint layer, and is usually caused by poor painting quality, contamination on the painting substrate, or deterioration by aging. It is difficult to detect with the conventional thickness meter, however [2]. Thus there is considerable need for terahertz nondestructive inspection of the paint-off area for marine protective coating.

Due to the strong reflection of the metal substrate, it commonly adopts the reflection mode of a TPI system. Usually, pulse echo reflection techniques are used for multilayer sample detection and a "peakfinding" method is used to analyze the time-domain terahertz waveforms. In TPI reflection geometry systems, the measured successive echo pulse at time domain is considered to be the convolution between the system pulse response and the raw reflected tissue signal. Thereby to characterize the inherent sample impulse functions and corresponding spectroscopic properties, the pulses reflected off the samples are routinely analyzed through a numerical Fourier deconvolution operation [11-12]. This process produces the pulse response function with a sharp spike at a time delay corresponding to the position of each reflecting interface of sample. A bandpass filter is typically incorporated into the inverse filtering to suppress the noise, such as a Gaussian filter[13-14] or double Gaussian filter[15-16], which can result in over-smoothing due to the cut-off frequency of the filter, however [17]. Ferguson group and Yang Chen group coupled the wavelet denoising with Wiener deconvolution to improve the resolution of the impulse response function remove, respectively. The former performed discrete wavelet transform (DWT) de-noising before Wiener deconvolution process and the later employed stationary wavelet 
shrinkage on the impulse function after Wiener deconvolution [18-23]. However, the Wiener filter not only suppresses the high-frequency noise but also removes the high-frequency components that represent the signals useful features [24]. The methods were based on the premise of wide-sense stationary process and white noise. It needs a priori knowledge of power spectrum of distorted wavelet as well as the noise characteristics. Only in the high signal-to-noise ratio (SNR) situation which is hard to meet in most engineering application, it could get optimal deconvolution results [25]. There were some studies focused on the signal spectrum characteristics of the impulse function, such as fixed-point iteration [26] and window function method [27] and so on. A few improved deconvolution methods were also proposed so far, such as total variation deconvolution [28], sparse deconvolution [29]. Very good and detailed technical reviews on TPI data processing algorithms based on deconvolution were published by Pickwell-MacPherson E [30].

In all, those deconvolution based processing methods are robust and intuitive and lead to the loss of useful information of signal, such as no longer able to resolve time domain features in the impulse response function and have a detection limit[23]. This was not good for the extraction of the faint feature, which was usually produced by the interface between two different medium layers with very similar refractive indices or caused by micro defects beneath the coating, and was not obvious in time-domain signal and hard to be located through the deconvolution based method.

Some scholars proposed a few methods about extracting the structural information from TPI raw signal without deconvolution process. T. Yasuda and T. Yasui employed multiple-regression analysis method to separate superposed $\mathrm{THz}$ echo pulses in place of numerical Fourier deconvolution in their THz paint meter [17]. T. Iwata proposed a modified partial-least-squares-1 method to TPI data for predicting the thickness of a single-layered paint film. However, they dispended on the parameters fitting or need to build a calibration model, both of which are not suitable for embedded defect detection [31]. 
T. Chady and P. Lopato adopt harmonic analysis method on TPI data for defects detection of glass-fiber reinforced composites [32]. D. J. Roth extracted power spectral density of TPI signal for foam inspection of the shuttle external tank [33]. Both the methods processed the results of discrete Fourier transform of obtained time sequences of signals, which can only represent the average distribution of signal power spectrum, and does not give the signal's time-frequency characteristics which could provide more localized features.

In the present work, we proposed an approach to extract the structure feature in marine protective coating by the combination of Hilbert transform (HT) and wavelet transform modulus maximum (WTMM) method. The samples with different coating structures were detected by an experimental TPI system and the corresponding FDTD-based models were also established. The occurrence of different paint-off defects (detachment or paint bulge) beneath coating was also investigated. Both the experimental and simulated TPI detected data was collected and then preprocessed by the proposed approach. The processing results were then used for the feature extraction of the coating structure.

\section{Signal processing for terahertz testing of marine protective coatings}

The protective coating system generally consist several layers of paint with different materials and it can be taken as a multilayered medium. Fig. 1 showed the diagram of a terahertz pulse interacts with marine protective coating on a reflective (i.e., metal) substrate. The $\mathrm{THz}$ pulse was incident on the sample surface and the $\mathrm{THz}$ pulse will be reflected back whenever there is a change in the refractive index or the optical absorption coefficient of the material (caused by either chemical or structural changes in the medium). The information on the chemical and physical properties of each layer was thus encoded into the reflected $\mathrm{THz}$ waveform; as a result, the structural information of a sample can be ultimately extracted by analysis of the recorded $\mathrm{THz}$ wave in the time domain. 


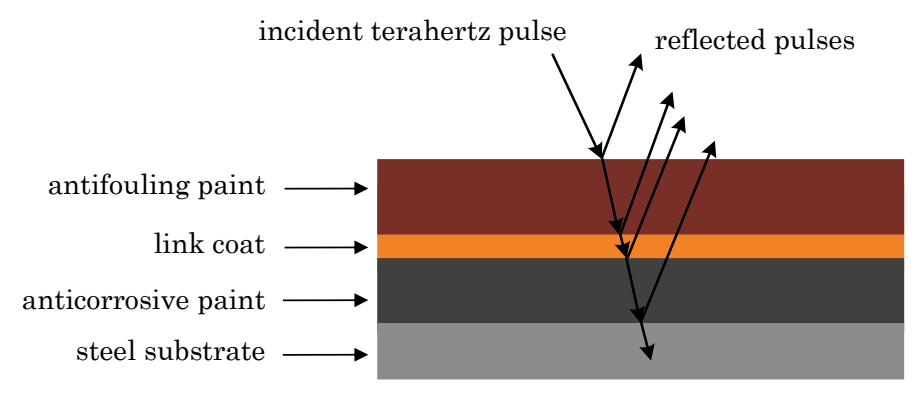

Fig. 1 The diagram of a terahertz pulse interacts with marine protective coating

A typical terahertz detected signal of a marine protective coating system in the time domain contained several echo caused by interfaces between air/surface of paint, paint/steel substrate and other internal interface between two different paints. Normally, the obtained data would affected by random noise due to material scattering as well as other background noise. When a defect exists inside the structure, there would be an abrupt variation in the signal. Even though the amplitude of the abrupt signal caused by the micro defect is smaller than noise and is not obvious in the time domain, but generally speaking, it varies drastically with noise [34]. This section aimed to extract the faint structure feature which was submerged with the noise. Fig. 2 showed the block diagram of signal processing for terahertz detected signal. Firstly, the TPI detected signal were preprocessed using the HT technology, and subsequently stationary wavelet transform (SWT) was applied to decompose the HT envelop into lowand high-frequency sub bands, which refer to the SWT approximation coefficients and detail coefficients, respectively. To overcome the difficulty in separating the useful signal which was submerged with the random noise, the modulus maxima of the detail coefficients were calculated and processed by the alternate projection algorithm to obtain new detail coefficients, which were then reconstructed with the approximation coefficients to produce a new signal. According to the relationship between WTMM and signal singularity, the new modulus maximal line of this new signal's approximation coefficients were employed for the feature extraction of the coating structure, especially for the defect localization. The detailed theory are discussed as follows. 


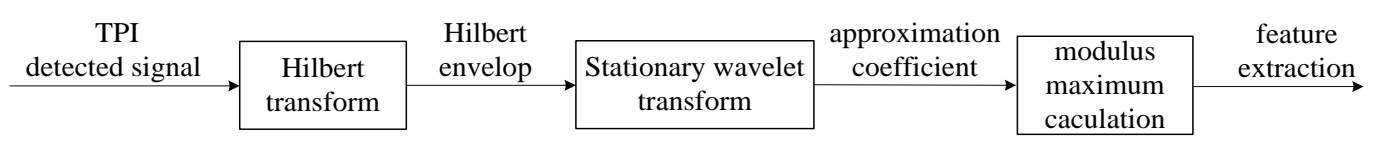

Fig.2 Block diagram of signal processing for terahertz detected signal

\subsection{Hilbert transform}

The Hilbert transform enables to create an artificial complex signal from the input signal. Here, the input signal is the terahertz detected signal $f(t)$. Its Hilbert transform is defined as [35]:

$$
H(t)=\frac{1}{\pi} \int_{-\infty}^{+\infty} f\left(t^{\prime}\right) /\left(x-x^{\prime}\right) \mathrm{dt}^{\prime}
$$

The Hilbert transform performs a $90^{\circ}$ phase shift or quadrature filter. Accroding to this characteristic, the analytic expression of the signal $f(x)$ can be constructed as

$$
Z(t)=f(t)+i H(t)=e(t) e^{i \phi(t)}
$$

whose real part is the original signal $f(t)$ itself and imaginary part is its Hilbert transform $H(t)$. The expression of the magnitude and instantaneous phase $\phi(t)$ can be derived as

$$
e(t)=\sqrt{f^{2}(t)+H^{2}(t)}, \quad \phi(t)=\arctan (H(t) / f(t))
$$

where $e(t)$ is called as Hilbert envelope. The Hilbert transform represents the instantaneous characteristics of wave signal and energy distribution in the time domain. It doesn't change the domain of signal, so the signal still in the time domain. The analytic signal of the real part excludes the negative frequency components and will not cause any loss of information. Geometrically, the Hilbert envelope means an integral curve which determines a singular position of the initial function [36]. Aim to extract the structure feature more clear and intuitive, we utilized Hilbert envelope of terahertz detected signal for further processing.

\subsection{Wavelet Transform Modulus Maximum (WTMM) for Singularity Detection}

The Hilbert envelop of the terahertz signal is still very coarse and needs further processing. When a structure has a flaw or defect, it would introduce some sorts of singularities to the signal. Most important 
information of a signal is carried by the position and the value of the local modulus maximal of the wavelet transform. WTMM method has been employed to extract explicit important features of images, as these features carry the information of sharp signal transitions and singularities [37]. In the field of signal processing, the information carried by WTMM is used to detect singularities, to eliminate noise and to reconstruct signals. In other words, because of the relation between WTMM and the local singularities, the signal can be represented and analysed through the local modulus maximal of the wavelet transform. In this study we employed WTMM with SWT as a means to detect singularities and to extract the structure feature for terahertz signal processing.

\subsubsection{Stationary Wavelet Transform (SWT)}

The SWT method is a wavelet transform algorithm designed to overcome the lack of translation invariance of the discrete wavelet transform (DWT). Translation- invariance is achieved by removing the down samplers and up samplers in the DWT and up sampling the filter coefficients by a factor of $2^{(j-}$ 1) in the $j$ th level of the algorithm, which makes SWT as a redundant transform and provides a more accurate estimate of the variances at each scale and facilitates the identification of salient features in a signal, especially for recognizing noise or signal rupture, such as the weak feature caused by micro defect. The SWT of the original data is not decimated, that is the size of the SWT data does not diminish after the transform, also known as undecimated wavelet transform [38-39]. The more detail on SWT can be founded in ref [40]. Fig. 3 shows a typical SWT decomposition procedure. $a_{1}, a_{2}, \cdots, a_{j}$ are the SWT approximation coefficients for decomposition level of $1,2, \cdots, j$, whist $d_{1}, d_{2}, \cdots, d_{j}$ are the SWT detail coefficients for decomposition level of $1,2, \cdots, j$. SWT makes that the existing local features of the data are not suppressed by averaging but observed in their natural position and extension. 


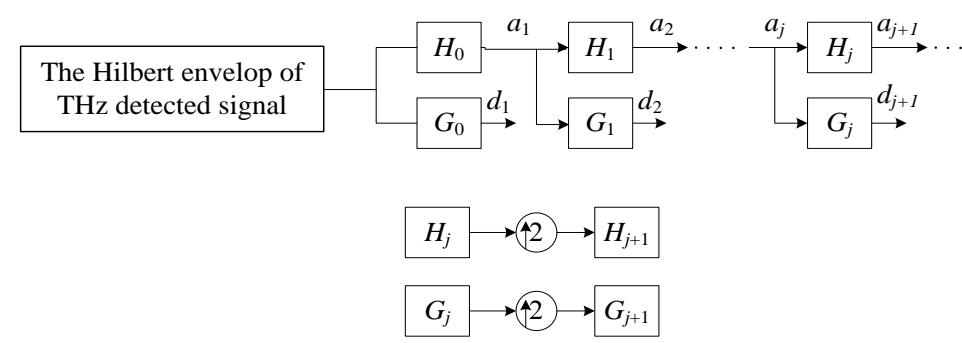

Fig. 3. SWT decomposition of Hilbert envelop of THz detected signal

\subsubsection{Singularity Detection based on WTMM}

WTMM reflects the properties of sharp signal transitions and singularities based on the fact that the wavelet transform of a signal will take its modulus maximum at its singular point in transform domain. This section would review the detection and characterization from the modulus maxima of a wavelet [41].

Assume the scaling function $\varphi(t)$ is a smooth function, whose integral is non-zero, that is, $\int_{-\infty}^{+\infty} \varphi(t) \mathrm{d} t=1$ and $\varphi(t)=\mathrm{O}\left(1 /\left(1+t^{2}\right)\right)$. Let $\psi(t)$ is the mother wavelet that has compact support, has $n$ vanishing moments, and is $n$ times continuously differentiable. When performing wavelet singularity analysis, the number of vanishing moments is very important, as it provides an upper bound measurement for singularity characterization.

$$
\psi^{1}(t)=\mathrm{d} \varphi(t) / \mathrm{d} t, \quad \psi^{2}(t)=\mathrm{d}^{2} \varphi(t) / \mathrm{d} t^{2}
$$

Introduce the scale factor $s$ to the function $\varphi(t)$, and let $\varphi_{s}(t)=\frac{1}{s} \varphi(t / s)$. When processing the terahertz signal in this paper, the Hilbert envelop $e(t)$ obtained above was used for wavelet transform. Let $e(t)$ is a finite-energy function, that is, $e(t) \in L^{2}(R)$. The wavelet transform of $e(t)$ defined with $\psi(t)$ is given by

$$
W_{s} e(t)=e(t) \psi_{s}(t)=\frac{1}{s} \int_{-\infty}^{+\infty} \psi((t-\tau) / s) \mathrm{d} \tau
$$

By combining the definitions of $\psi(t), \varphi_{\mathrm{s}}(t)$ and Eq. (6), we then obtain

$$
\begin{aligned}
& W_{s}^{1} e(t)=W^{1} e(s, t)=e(t) * \psi_{s}^{1}(t)=e(t) * s \cdot \mathrm{d} \varphi_{s}(t) / \mathrm{d} t=s \cdot \mathrm{d}\left(e(t) * \varphi_{s}(t)\right) / \mathrm{d} t \\
& W_{s}^{2} e(t)=W^{2} e(s, t)=e(t) * \psi_{s}^{2}(t)=e(t) * s^{2} \cdot \mathrm{d}^{2} \varphi_{s}(t) / \mathrm{d} t^{2}=s^{2} \cdot \mathrm{d}^{2}\left(e(t) * \varphi_{s}(t)\right) / \mathrm{d} t^{2}
\end{aligned}
$$


where “*” represents convolution procedure, the wavelet transforms $\mathrm{W}_{\mathrm{s}}{ }^{1} e(t)$ and $\mathrm{W}_{\mathrm{s}}^{2} e(t)$ represent are the first derivatives and the second derivatives of $e(t)$ smoothed by $\varphi_{\mathrm{s}}(t)$ at the scale $s$, respectively. It can be verified that the corresponding relations exist between the singularities of $e(t)$ and the local modulus maximal of $\mathrm{W}_{\mathrm{s}}{ }^{1} e(t)$ and $\mathrm{W}_{\mathrm{s}}^{2} e(t)$.

So an inflection point in a signal can be either a maximum or a minimum of the absolute value of the first derivative $\mathrm{W}_{\mathrm{s}}^{1} e(t)$, which refers to the local extreme approach. The former corresponds to the sharp variation points of the smoothed function, whereas the later corresponds to the slow variations [41]. On the other hand, both of the maximum and minimum have the property of zero-crossing in the second derivation $\mathrm{W}_{\mathrm{s}}^{2} e(t)$. That is, one can detect the mutation either by the local extreme approach in $\mathrm{W}_{\mathrm{s}}{ }^{1} e(t)$ or by locating the zero-crossings of $\mathrm{W}_{\mathrm{s}}^{2} e(t)$. It has been verified that the local extreme approach in $\mathrm{W}_{\mathrm{s}}{ }^{1}$ $e(t)$ is better than that of detecting the zero-crossings of $\mathrm{W}_{\mathrm{s}}^{2} e(t)$ [42]. So in the present work, the first derivative $\mathrm{W}_{\mathrm{s}}{ }^{1} e(t)$ was calculated and employed for terahertz signal singularity detecting. The definition of the local modulus maximal of the wavelet transform is: At scale $s_{i}$, the point $\left(s_{i}, t_{0}\right)$ of the first derivative $\mathrm{W}_{\mathrm{s}}{ }^{1} e(t)$ was called as the local modulus maximal, if all points belong to either the right or the left neighborhood of $t_{0},\left|\mathrm{~W}_{s i}{ }^{1} e(t)\right| \leq\left|\mathrm{W}_{s i}{ }^{1} e\left(t_{0}\right)\right|$. The local maxima of the wavelet transform modulus provide enough information to detect and analyze all discontinuities inside signal. For our signal processing techniques in the work, after the Hilbert envelop decomposed by SWT, the modulus maxima of the approximation coefficients were needed for feature extraction. Generally, sturcture features and noise would mix together in signal and lead to the fact that there are a lot of nonzero coefficients with small magnitude in the wavelet coefficients. If the amplitude of the abrupt signal caused by the micro defect is smaller than one of noise, it is hard to exactly determine the location and needs to separate the noise out of the useful signal. Based on their different singularities, the modulus maximum of the useful signal increases and the modulus maximum of noise decreases with the augment of wavelet scale [43]. 
Due to the noise mainly distributes in the high frequency part, the multiplication of the detailed coefficients in some successive decomposition levels can diminish background noise and reinforce the presence of mutation in the recorded data. Therefore in the present work, the modulus maxima of the detail coefficient was calculated and processed by the alternate projection algorithm to obtain a new detail coefficient [24]. Subsequently, the new de-noised detail coefficient was reconstructed with the approximation coefficient to generate a new signal. This process can greatly reduce noise while preserving most of the important features of the signal. The new modulus maximal line of the new signal's approximation coefficient became clearer and more recognizable in transform domain and it was satisfied for providing the information of marine coating structure for internal defect characterization.

\section{Results and discussions}

\subsection{Materials and methods}

Generally, different areas of a ship require specific anti-corrosive coating. Steel corrosion at and around the waterline is of considerable practical interest for ships. The coating system in that region is required to prevent corrosion and fouling, which is achieved by using an anticorrosive paint and an antifouling paint [44]. The specific surface treatment method involves the application of organic coatings that exhibit high cavitation resistance and good erosion resistance [45], thus most paints are based on organic solvents. In our experiments, the samples consisted of a steel panel coated with layers of protective paint to mimic the marine protective coating below the waterline as: Two layers of anticorrosive primer paint (Jotaprime 500, which was consisted of resins, polyurethane etc.) were uniformly sprayed on the metal substrate; it was followed by one layer of so - called "link coat" (5BP Safeguard Plus) which provides a good connection with both the anticorrosive primer paint and then the self-polishing antifouling paint (SeaMate) which were sprayed lastly by three layers and were used to prevent attachment and growth of marine organisms. There were four samples (10mm x 10mm) with 
different paint layer thickness, as shown in Table 1. Noted here that "sprayed layer order" in the table means the painting order of coatings.

Table 1. Detailed information of marine protective coating structures for TPI detected

\begin{tabular}{|c|c|c|c|c|}
\hline Sample name & Sprayed layer order & Paint sprayed & Function & $\begin{array}{l}\text { Measured average } \\
\text { thickness (um) }\end{array}$ \\
\hline \multirow{6}{*}{ Sample1 } & layer 1 & 500 Jotaprime 500 & anticorrosive paint & 145 \\
\hline & layer 2 & 500 Jotaprime 500 & anticorrosive paint & 142 \\
\hline & layer 3 & 5BP Safeguard Plus & link coat & 75 \\
\hline & layer 4 & SeaMate & antifouling paint & 108 \\
\hline & layer 5 & SeaMate & antifouling paint & 105 \\
\hline & layer 6 & SeaMate & antifouling paint & 101 \\
\hline \multirow{6}{*}{ Sample2 } & layer 1 & 500 Jotaprime 500 & anticorrosive paint & 186 \\
\hline & layer 2 & 500 Jotaprime 500 & anticorrosive paint & 160 \\
\hline & layer 3 & 5BP Safeguard Plus & link coat & 95 \\
\hline & layer 4 & SeaMate & antifouling paint & 126 \\
\hline & layer 5 & SeaMate & antifouling paint & 135 \\
\hline & layer 6 & SeaMate & antifouling paint & 140 \\
\hline \multirow{6}{*}{ Sample3 } & layer 1 & 500 Jotaprime 500 & anticorrosive paint & 205 \\
\hline & layer 2 & 500 Jotaprime 500 & anticorrosive paint & 195 \\
\hline & layer 3 & 5BP Safeguard Plus & link coat & 95 \\
\hline & layer 4 & SeaMate & antifouling paint & 153 \\
\hline & layer 5 & SeaMate & antifouling paint & 161 \\
\hline & layer 6 & SeaMate & antifouling paint & 174 \\
\hline \multirow{6}{*}{ Sample4 } & layer 1 & 500 Jotaprime 500 & anticorrosive paint & 169 \\
\hline & layer 2 & 500 Jotaprime 500 & anticorrosive paint & 160 \\
\hline & layer 3 & 5BP Safeguard Plus & link coat & 92 \\
\hline & layer 4 & SeaMate & antifouling paint & 135 \\
\hline & layer 5 & SeaMate & antifouling paint & 130 \\
\hline & layer 6 & SeaMate & antifouling paint & 132 \\
\hline
\end{tabular}

For all TPI measurements, a TAS7500IM system (Advantest Corporation, Japan) was used with reflected mode. The terahertz radiation was broadband, covering a spectral range of 75-300um (0.1-4 $\mathrm{THz}$ ). An example of time domain terahertz-wave signals reflected back from a metal mirror and a 
coated sample (sample1) was shown in Fig. 4. The reference signal and the measurement signal denoted reflection signals from the metal mirror and coated sample, respectively. The signal had been normalized to the maximum reference signal.

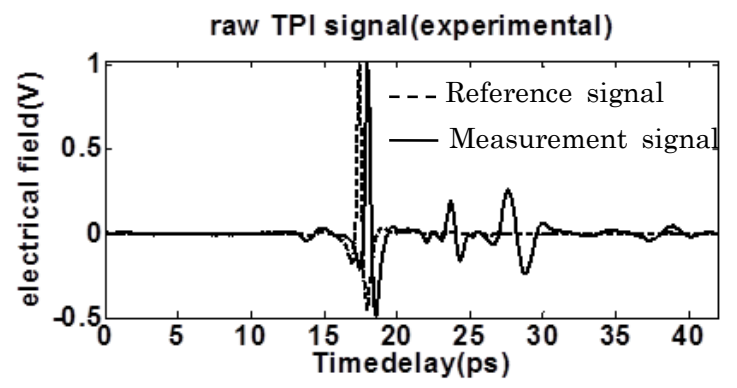

Fig. 4. Time domain terahertz-wave signals reflected back from the metal mirror and a coated sample (sample1)

Usually, THz signal feature in frequency domain was extracted to help characterizing materials, such as single layer coatings [12] and paper basis weight [46]. Fig. 5(a) and (b) showed the amplitude spectrum and phase spectrum of the detected THz signals for four samples which were listed in Table1. Furthermore, phase shift, which has been verified to be associated with sample thickness, can be deduced from phase spectra of sample signal and reference signal [46]. Fig. 5(c) showed the phase shift curves for the four samples. 
(a)

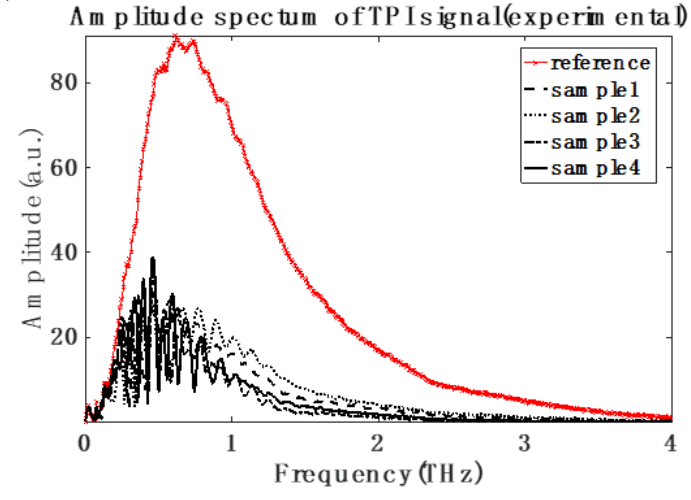

(b)

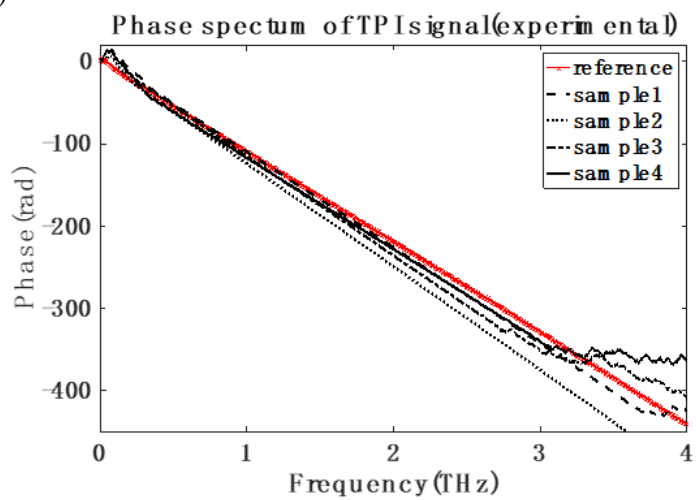

(c)

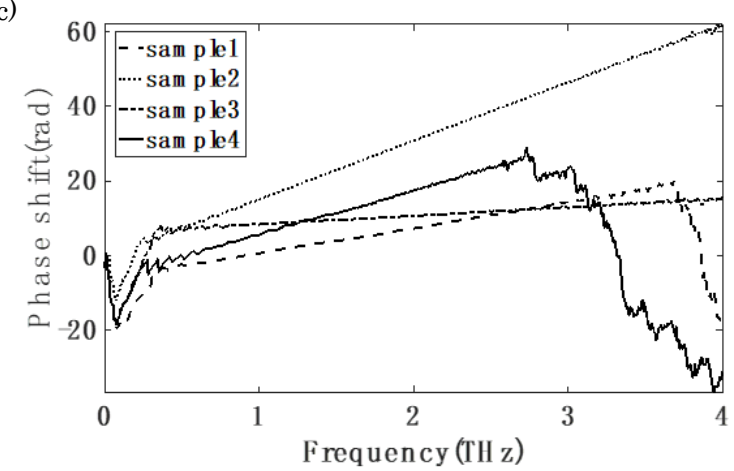

Fig. 5. Signal feature in frequency domain of detected terahertz signals: (a) Amplitude spectrums of reference signal and four samples signals; (b) the corresponding phase spectrums; (c) The phase shift curves for the four samples.

As can be seen from Fig.5 (a) and (b), although there were some differences in amplitude and phase spectrums between the four samples, it was hard to get directly the relationship between the amplitudes spectrums and coating thicknesses of the multilayered structures. Moreover, the phase shift method was suitable for single layer structure characterization [46], however, it is difficult to provide directly the relationship between phase shift and the four multilayered coating structures.

Furthermore, theoretical model based on the FDTD method for terahertz detection of marine protective coating was also investigated. It has been demonstrated that FDTD model could be capable of calculating the propagation and reflection of $\mathrm{THz}$ radiation from a multilayered flat or curved structures [47]. More detail on FDTD simulation of terahertz wave interacting with multilayered medium can be founded in our precious work [47-48]. In our model, a cylinder plate of $10 \mathrm{~mm} * 10 \mathrm{~mm}$ size with 
$0.3 \mathrm{~mm}$ thickness was taken as steel substrate, and the paint layers were successively coated on the steel substrate to mimic the coating samples. The protective coating was treated as a linear isotropic homogeneous layer without magnetic field loss. For simplicity, the dispersive properties and the specific conductivity of coatings were negligible as well [7]. From Fig.4, the refractive index of antifouling paint can be obtained through the amplitude of the reference signal and sample signal, antifouling paint layers thickness and time delay [49]. It was calculated as 1.98. Besides, the value of reflection peak caused by the interface between two different kinds of material was associated with their difference in refractive index, thus it can be referred that the refractive index of link coat is little smaller than that of antifouling paint and much smaller than that anticorrosive paint. In simulation, the refractive indices of paint layers were set as: $n_{\text {antifouling paint }}=1.98, n_{\text {link coat }}=1.8, n_{\text {anticorrosive paint }}=2.2$. All FDTD simulations here were performed using the Remcom XFDTD commercial software package. The incident terahertz plane wave was set perpendicular to the sample surface and a Gaussian profile of the amplitude distribution of the E-field was initiated in the incident plane with peak amplitude of $1 \mathrm{~V} / \mathrm{m}$. The electric fields of the reflected pulses caused by the internal interfaces were recorded and represented the raw terahertz detected signal. In order to mimic the background noise of the real experiments, Gaussian white noise was added to the obtained data in a SNR of $32 \mathrm{~dB}$ [21].

\subsection{Feature extraction for multilayered coating structures}

Fig.6 (a-1) Fig.9 (a-1) were experimental time domain terahertz-wave signals reflected back from the four coated samples, and Fig.6 (b-1) Fig.9 (b-1) were the corresponding simulated TPI signals with Gaussian white noise. The origin of the horizontal axis corresponds to the surfaces of the metal mirror and sample. The reflected pulses caused by interfaces between different paint layers can be seen either from the simulated signals or the experimental signal. The oscillation after the reflection from the coating/steel interface was caused by multiple reflections inside the sample [50]. The time-domain 
spacing of the sub-peaks can simply be converted to depth information [51]. Fig.6(a-2) Fig.9 (a-2) and Fig.6 (b-2) Fig.9 (b-2) were the impulse functions obtained by Gaussian deconvolution for experimental and simulated TPI signals, respectively. As can be seen from impulse functions, the refractive indices of paint medium set for simulation were consistent with the actual material. Besides, although the noise was mostly suppressed in deconvolution process it is still hard to exactly determine the each interface position due to over-smoothing.

Both the experimental and simulated raw TPI signal was first preprocessed by the HT technology to derive the Hilbert envelop, which retained the mutations and singular points of the original signal. The db1 wavelet was used and the three layer SWT decomposition and reconstruction was carried out to Hilbert envelops to get new signals. Subsequently the modulus maximum of the new signals' SWT approximation coefficients was obtained and shown in Fig.6 (a-3) Fig.9(a-3) and Fig.6 (b-3) Fig.9 (b-3). From the figures, all the paint layer interfaces can be clearly identified and characterized.

It should be noted that, in the real application of TPI testing of protective coatings, several sources of random and systematic errors exist throughout the measurement process, which can affect the accuracy and uncertainty of the test. These sources are, for instance, signal noise, sample misalignment, thickness measurement variation, surface of layers uneven, etc [51]. Thus, the added Gaussian with noise in the simulated result could not accurately represent the actual interference in practice. Howevrer, it was often adopted for theoretical studies of THz test method[21]. Besides, the amplitude of reflection peak in simulation was different from the experimental test. For example, the dominant peak in experimental signals represented the signal reflected by air/coating interface, while the second largest peak referred to the signal reflected by coating/steel interface. However, the dominant peak in simulated signals represented the signal reflected by coating/steel interface for its strong reflection, while the second largest peak referred to the signal reflected by air/coating interface. That was mainly because the 
dispersive properties and the specific conductivity of coatings were negligible in simulation, which lead to that the wave reflected by coating/steel interface has great energy reservations. While it cannot be ignored for the actual material, which resulted in that the paint layers has reflected off lot of energy and the signal reflected by air/coating interface was much smaller. For structure feature extraction, it was little affected because the pulses between those two strong reflections were important and need to be focused on. Compared to the expensive TPI measurement, FDTD simulation could be cheaper and more flexible to mimic terahertz signals from multilayered samples.

$(\mathrm{a}-1)$

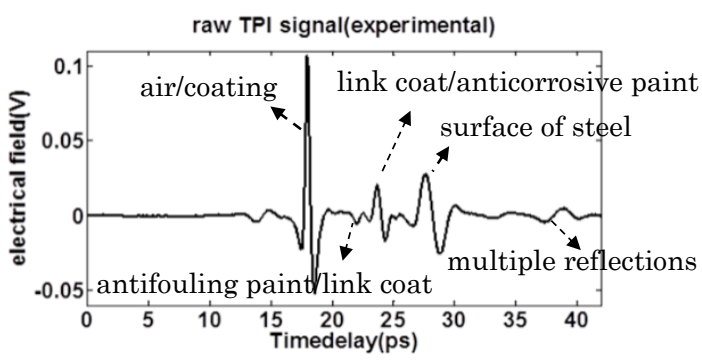

$(\mathrm{a}-2)$

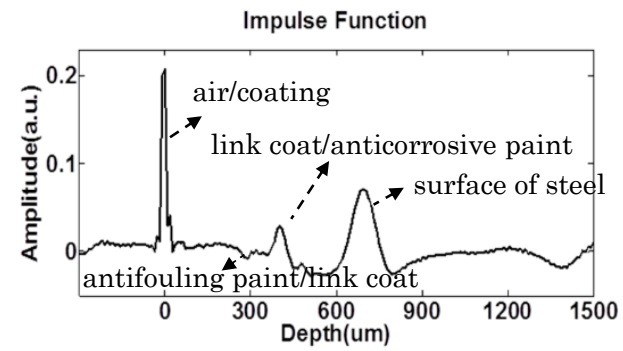

$(\mathrm{a}-3)$

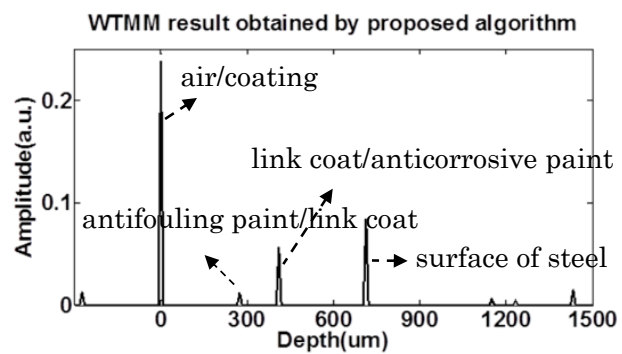

$(b-1)$

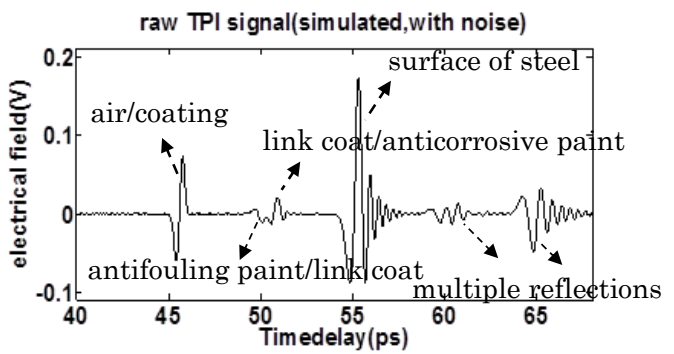

$(b-2)$

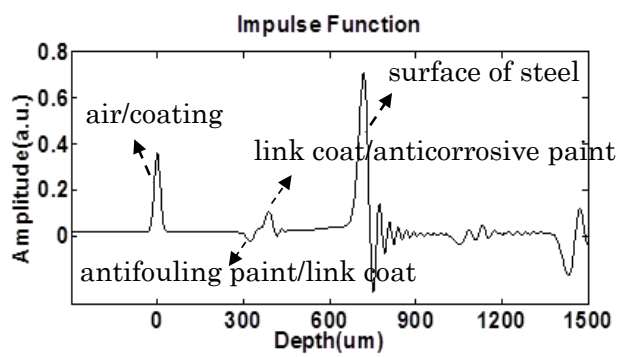

$(b-3)$ WTMM result obtained by proposed algorithm

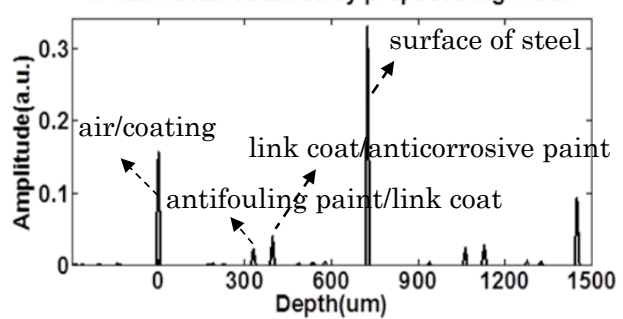

Fig. 6. TPI signal processing results for Sample1 
$(\mathrm{a}-1)$

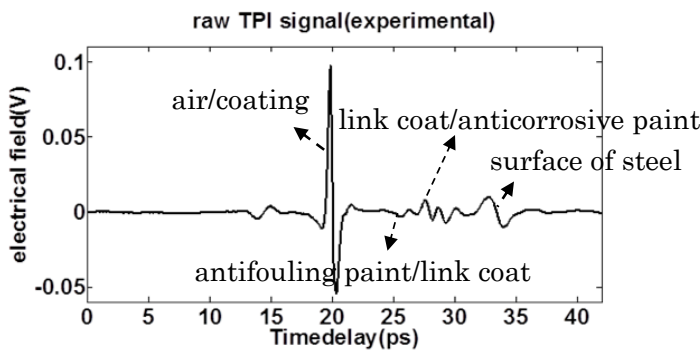

$(\mathrm{a}-2)$

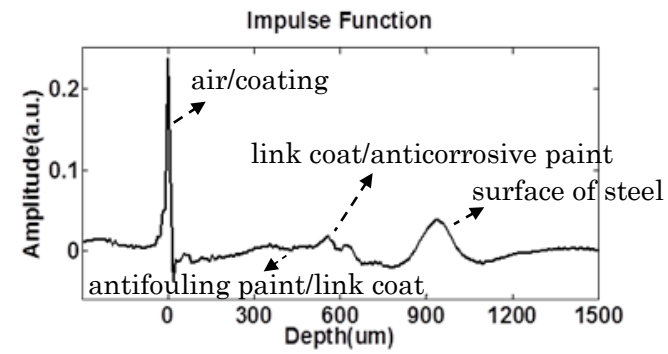

$(\mathrm{a}-3)$

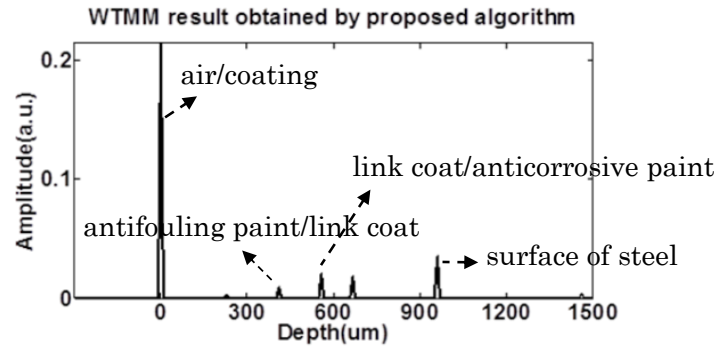

(b-1)

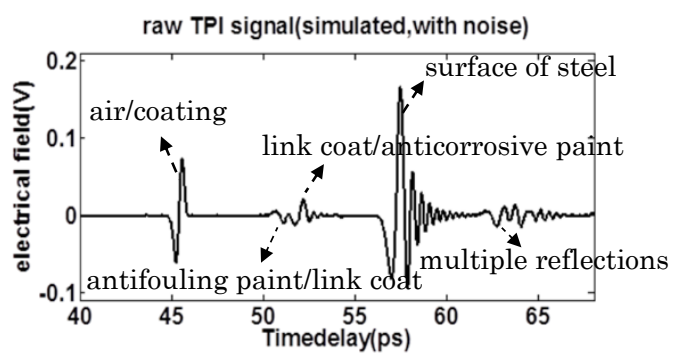

(b-2)

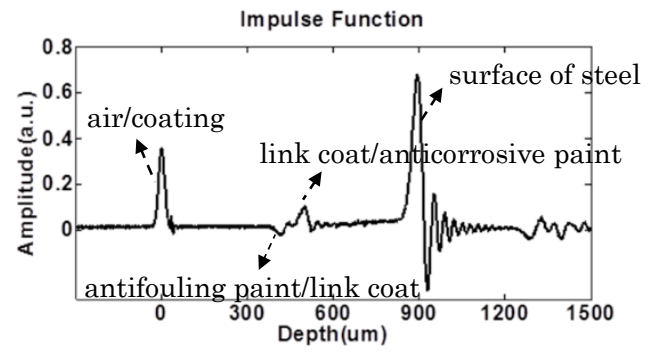

(b-3)

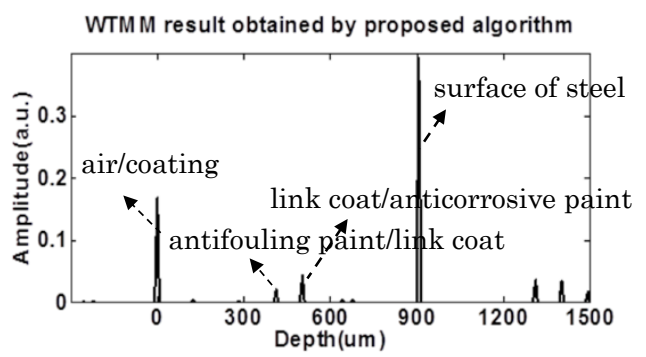

Fig. 7. TPI signal processing results for Sample2

$(\mathrm{a}-1)$

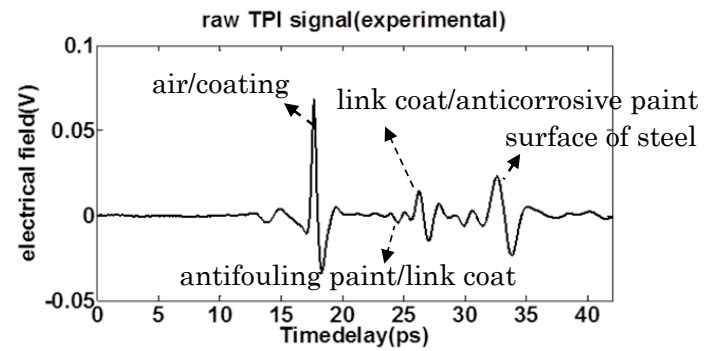

$(\mathrm{a}-2)$

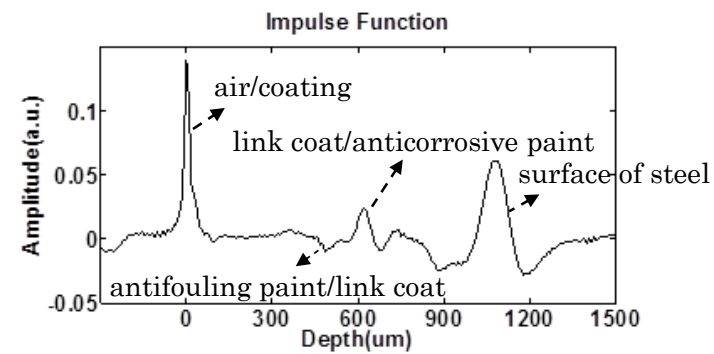

$(\mathrm{a}-3)$

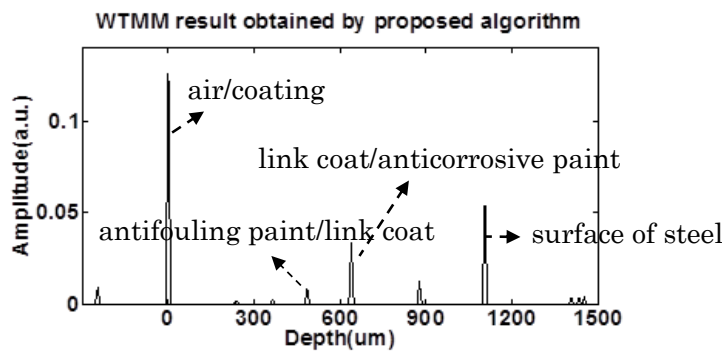

$(b-1)$

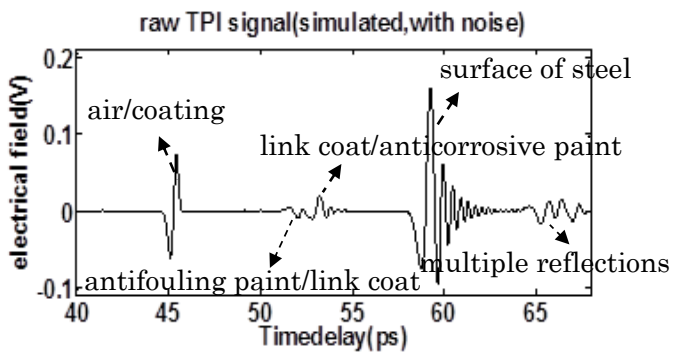

$(\mathrm{b}-2)$

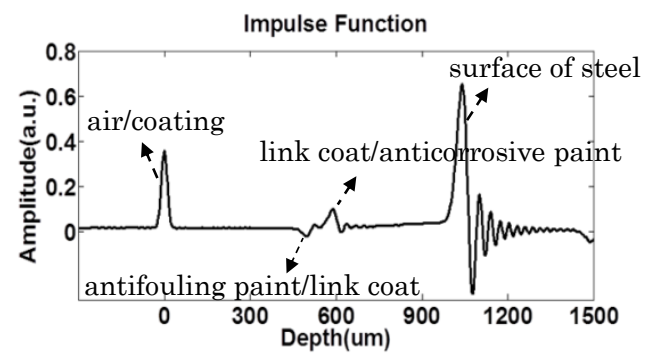

$(\mathrm{b}-3)$

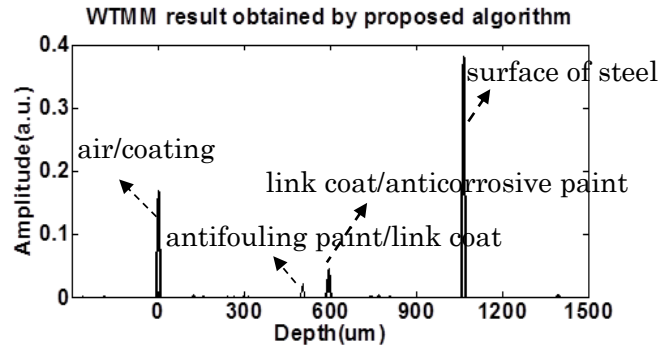

Fig. 8. TPI signal processing results for Sample3 
$(\mathrm{a}-1)$

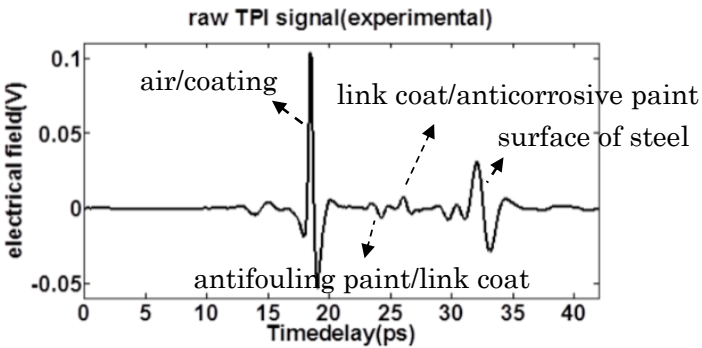

$(\mathrm{a}-2)$

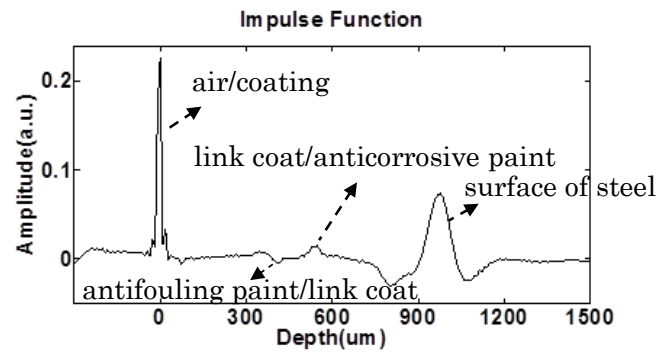

$(\mathrm{a}-3)$

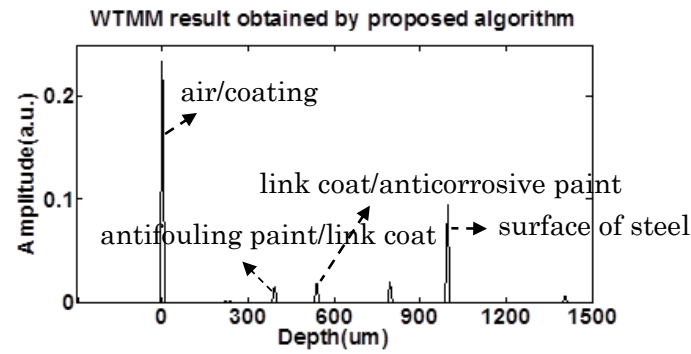

$(\mathrm{b}-1)$

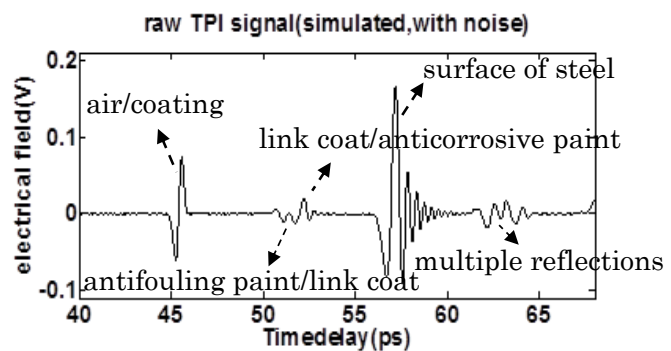

$(b-2)$

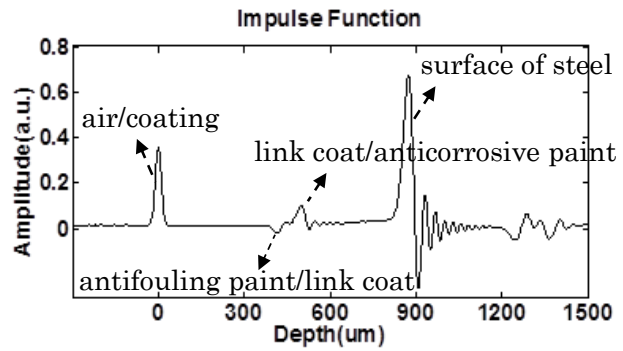

$(b-3)$

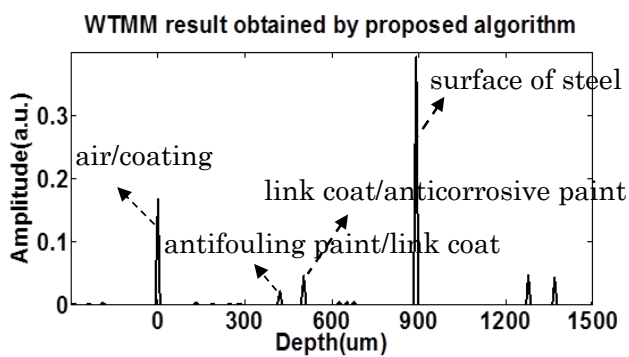

Fig. 9. TPI signal processing results for Sample4

\subsection{Feature extraction for marine coating with defects}

Defects inside the coating system will cause either chemical or structural changes and therefore cause a change in refractive index or absorbing coefficient of the material which would have unique signatures in the $\mathrm{THz}$ reflected signals. It can be detected and distinguished by terahertz radiation technology. The paint off defect beneath marine protective coating was investigated both by experiment and simulation in this paper. One situation was the paint layer detachment from the steel substrate which was shown as Fig.10 (a-1), another one was paint bulge defect (or bubble) which was shown as Fig.10 (a-1). The raw experimental TPI detected signals were shown as Fig.10 (a-2) Fig.11 (a-2). Fig.10 (b-1) Fig.11 (b1) was cross section profile of the corresponding simulated models to mimic the experimental sample. One defect of curved shape with $3.5 \mathrm{~mm}$ radius and $150 \mathrm{um}$ thickness was embedded between the coating and steel substrate representing detachment defect beneath coating. The second defect of curved shape 
with $3.5 \mathrm{~mm}$ radius and 350um thickness was embedded inside the antifouling layers with depth 100um from coating surface, representing paint bulge defect. The reflected terahertz waveforms were recorded and shown as Fig.10 (b-2) Fig.11 (b-2). The simulation was Fig.10 (a-3) Fig.11 (a-3) and Fig.10 (b3) Fig.11 (b-3) were the corresponding impulse functions obtained by Gaussian deconvolution for experimental and simulated TPI signals. The defect feature was hard to be characterized both in raw TPI signals and their impulse functions. Fig.9 Fig.10 (a-4) and (b-4) were the modulus maximum results processed by the proposed algorithm, respectively.

Compared to the samples without a paint-off defect (Fig.10 vs. Fig.6, Fig.11 vs. Fig.7), the defects can be identified and characterized. It was enough for defect localization and evaluation in the coating. Besides, compared to impulse functions obtained from the traditional deconvolution method, the structural change caused by paint-off defect in the marine protective coating can be easier and clearer detected by WTMM results obtained by the combination of Hilbert transform and WTMM method. It was able to provide much more detail information on defect beneath the coating, such as detachment of large areas where adhesion has failed, and so on. 
$(\mathrm{a}-1)$

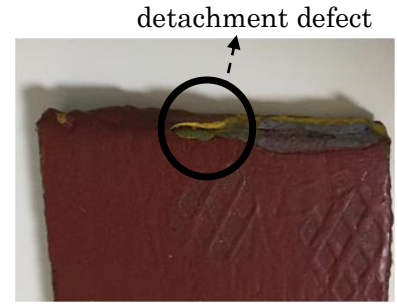

$(\mathrm{a}-2)$

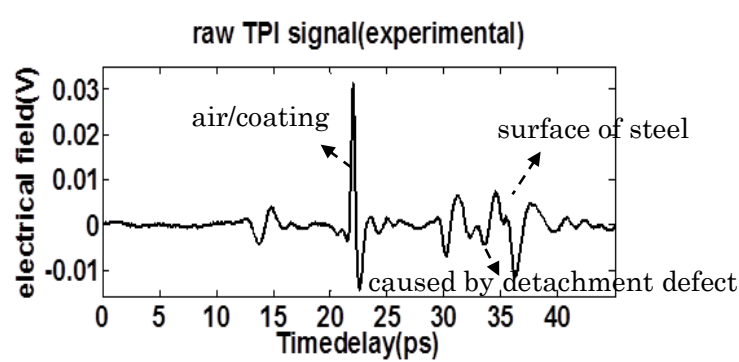

$(\mathrm{a}-3)$

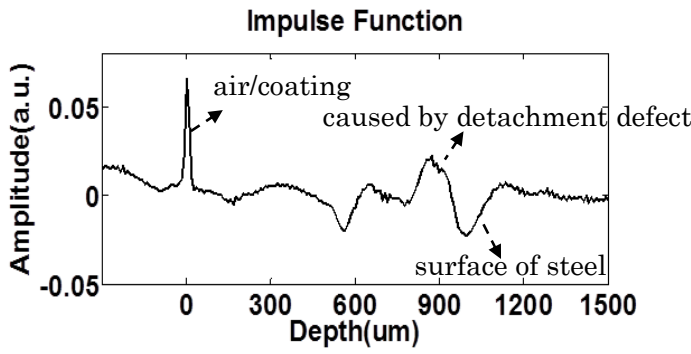

$(\mathrm{a}-4)$

WTMM result obtained by proposed algorithm

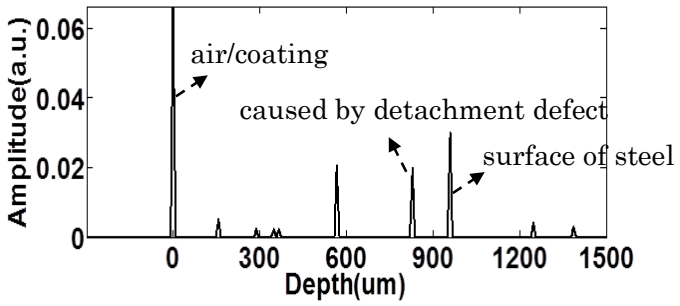

$(\mathrm{b}-1)$

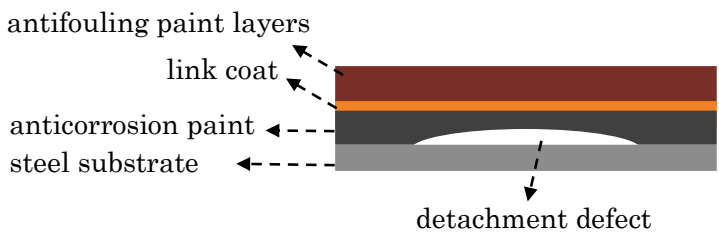

$(b-2)$

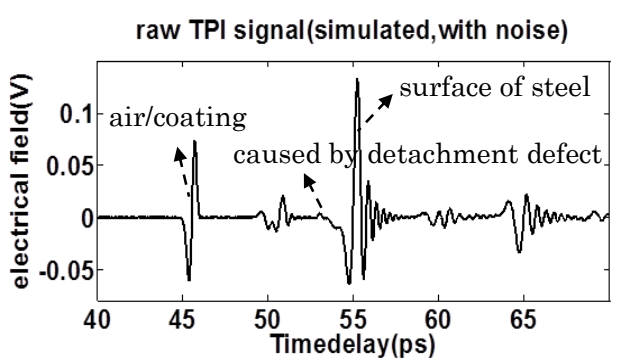

$(b-3)$

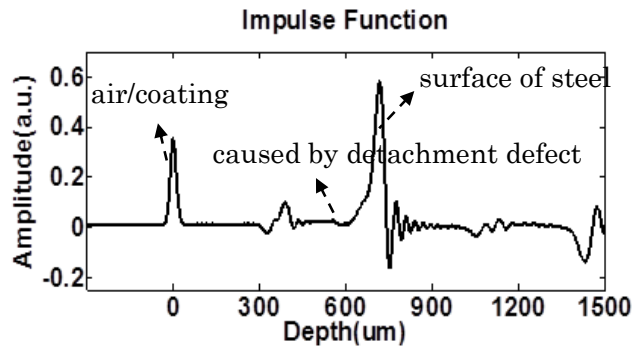

$(\mathrm{b}-4)$

WTMM result obtained by proposed algorithm

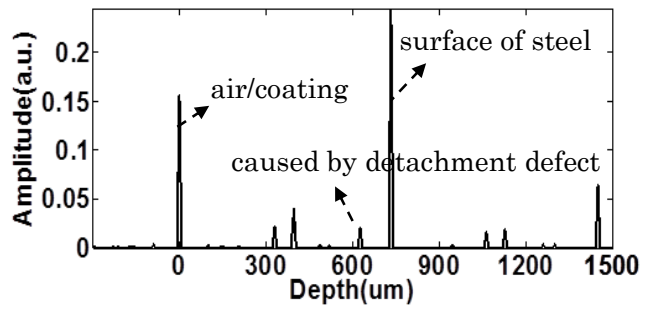

Fig. 10. TPI signal processing results for Sample1 with detachment defetct between coating and steel substrate 
$(\mathrm{a}-1)$

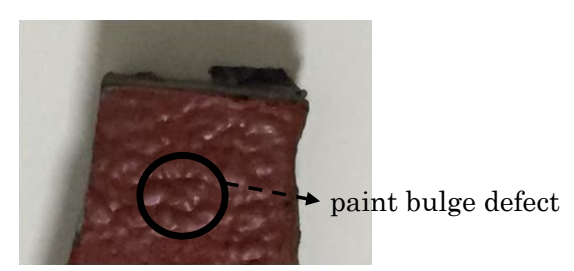

$(a-2)$

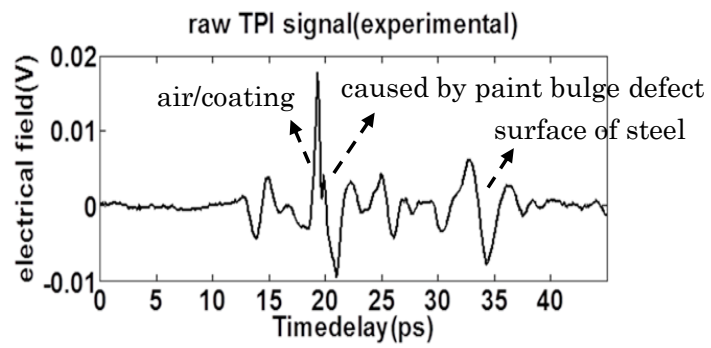

$(\mathrm{a}-3)$

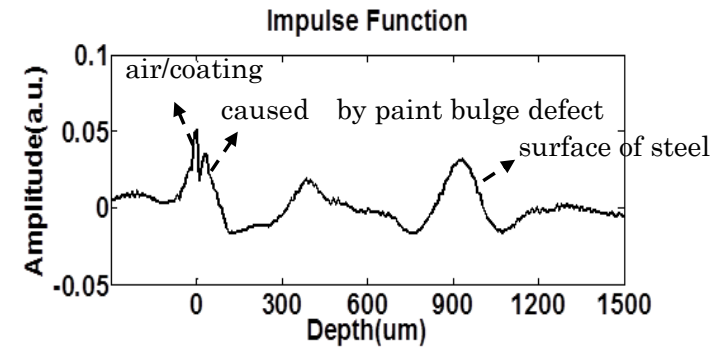

$(\mathrm{a}-4)$

WTMM result obtained by proposed algorithm

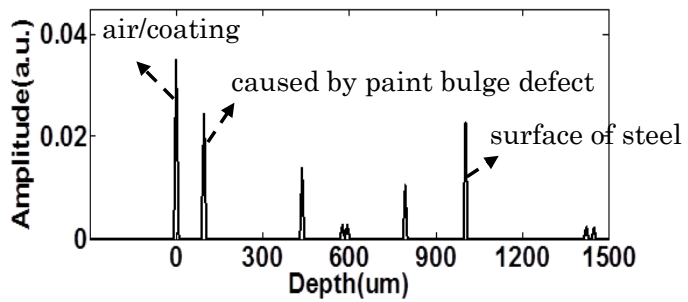

$(b-1)$

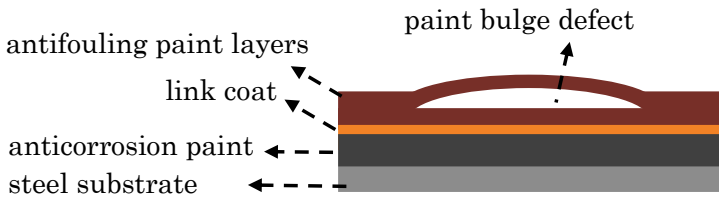

$(\mathrm{b}-2)$

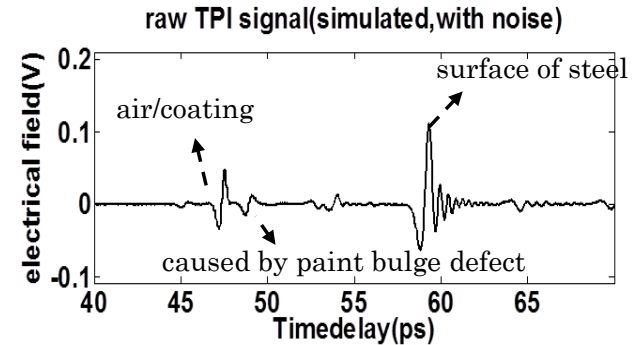

$(b-3)$

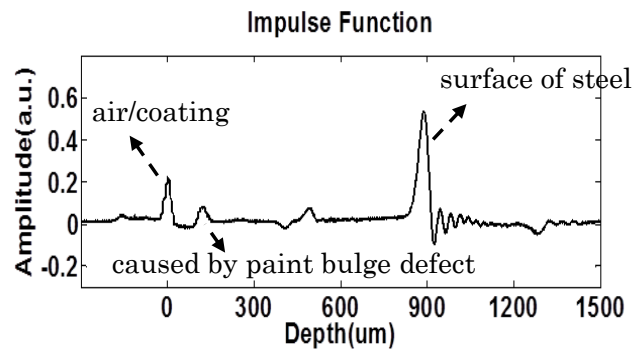

$(\mathrm{b}-4)$

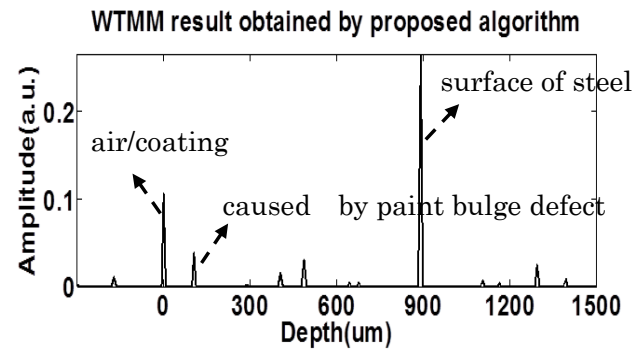

Fig. 11. TPI signal processing results for Sample2 with paint bulge defetct inside antifouling paint layers

\section{Conclusions}

In summary, the TPI data was preprocessed by the combination of Hilbert transform and wavelet transform modulus maximum method for defect feature extraction of marine protective coatings. The HT technology was used to derive the Hilbert envelop, which retained the mutations and singular points of the original signal and was then decomposed by SWT. The modulus maximum of the SWT approximation coefficients were used for defect feature extraction of the coating structure. Coating samples with different paint thickness and different paint-off defects were evaluated. Both experimental 
and simulated results demonstrated that TPI technology combined with the proposed new algorithm was an effective method for revealing hidden interface information and for localization of micro defect beneath coating, even when the background noise is present. The proposed method aimed at the structure feature extraction of multilayered marine protective coating, where it would facilitate the quality inspection of painting process and benefit the effective maintenance to avoid coating failure for marine protective coating. However, for the achievable depth resolution is generally constrained to behalf the coherent length of the terahertz pulse in a sample, it is hard to carry out the proposed method on thinner layer coating detection at the early stage of painting process. So far, there were some processing technologies to analyze the thinner coating such as multiple-regression analysis method, artificial neural networks predictive analysis method, and the improved feature extraction technology for small defects of marine protective coatings will be considered as the future work. We believe that the use of the proposed processing algorithms and $\mathrm{THz}$ imaging measurements will provide a powerful method for non-destructive testing and evaluation of marine protective coating.

\section{Acknowledgements}

We gratefully acknowledge support from the National Natural Science Foundation of China (51005077), the Fujian Provincial Excellent Young Scientist Fund (2014J07007), the Specialised Research Fund for the Doctoral Program of Higher Education, the Ministry of Education, P. R. China (20133514110008), the Ministry of Health, P.R. China (WKJ-FJ-27), the Education Department of Fujian Province (JA13180), the Fujian Provincial Natural Science Foundation (2016J01250, 2015J01234) and the Fujian Provincial Quality and Technical Supervision Bureau Project (FJQI2014008, FJQI2013024)..

\section{References}


1 Yun-Shik, L. 2008. Principles of Terahertz Science and Technology. Springer-Verlag, New York, pp. 159-170.

2 Yasui, T., Yasuda, T., Sawanaka, K. I., Araki, T., 2005. Terahertz paintmeter for noncontact monitoring of thickness and drying progress in paint film. Appl. Opt.,44, 6849-6856.

3 Izutani, Y., Akagi, M., Kitagishi, K., 2012. Measurements of Paint Thickness of Automobiles by using THz Time-Domain Spectroscopy 37th International Conference on Infrared, Millimeter, and Terahertz Waves (IRMMW-THz), , pp. 1-2.

4 Fukuchi, T., Fuse, N., Okada, M., Fujii, T., Mizuno, M., Fukunaga, K., 2013. Measurement of Refractive Index and Thickness of Topcoat of Thermal Barrier Coating by Reflection Measurement of Terahertz Waves. Electron. Comm. Jpn., $96(12), 37-45$.

5 Cook, D. J., Sharpe, S. J., Lee, S., Allen, M. G., 2007. Terahertz time domain measurements of marine paint thickness. Opt. Terahertz Sci. Tec., TuB5.

6 Cook, D. J., Lee, S., Sharpe, S. J., Allen, M. G., 2008. Accuracy and linearity of time-domain THz paint thickness measurements. Proc. of SPIE, Vol. 6893, 68930H.

7 Soares, C. G., Garbatov, Y., Zayed, A., Wang, G., 2009. Influence of environmental factors on corrosion of ship structures in marine atmosphere. Corros. Sci., 51: 2014-2026 .

8 Akpan, U. O., Koko, T. S., Ayyub, B., Dunbar, T. E., 2002.Risk assessment of aging ship hull structures in the presence of corrosion and fatigue. Mar. Struc., 15: 211-231.

9 Adey R. A., Baynham J., 2000. Design and optimisation of cathodic protection systems using computer simulation. CORROSION 2000, National Association of Corrosion Engineers.

10 Deardorff, J. R., 2009. Non-destructive testing for protective coatings-Implementing a lifetime corrosion prevention program. Met. Finish., 107: 31-39.

11 Pickwell, E., Cole, B. E., Fitzgerald, A. J., Pepper, M., Wallace, V. P., 2004. In vivo study of human skin using pulsed terahertz radiation. Phys. Med. Biol., 49(9), 1595.

12 Zhong S, Shen Y, Evans M J, et al., 2010. Neural Network-based non-destructive quantification of thin coating by terahertz 
pulsed imaging in the frequency domain. 35th International Conference on Infrared Millimeter and Terahertz Waves (IRMMW-THz). IEEE, 1-2.

13 Pickwell-MacPherson E., Wallace V. P., Cole B. E., Ali S., Longbottom C., Lynch R.J.M., Pepper M., 2007. A comparison of terahertz pulsed imaging with transmission microradiography for depth measurement of enamel demineralisation in vitro.

Caries Res., 41: 49-55.

14 Pickwell-MacPherson E, Huang S, Sun Y, et al., 2008. Terahertz image processing methods for biomedical applications. Annual International Conference of the IEEE Engineering in Medicine and Biology Society, 3751-3754.

15 Woodward R M, Cole B E, Wallace V P, et al., 2002. Terahertz pulse imaging in reflection geometry of human skin cancer and skin tissue. Physics in medicine and biology, 47(21): 3853.

16 Crawley D, Longbottom C, Wallace V P, et al., 2003. Three-dimensional terahertz pulse imaging of dental tissue. Journal of Biomedical Optics, 8(2): 303-307.

17 Yasuda T., Iwata T., Araki T., et al., 2007. Improvement of minimum paint film thickness for THz paint meters by multiple-regression analysis. Applied optics, 46(30): 7518-7526.

18 Ferguson B., Abbott D., 2001. De-noising techniques for terahertz responses of biological samples. Microelectronics Journal, 32(12): 943-953.

19 Ferguson B., Abbott D., 2001. Wavelet de-noising of optical terahertz pulse imaging data. Fluctuation and Noise Letters, 1(02): L65-L69.

20 Yin X., Ng B. W. H., Abbott D., et al., 2007. Application of auto regressive models of wavelet sub-bands for classifying terahertz pulse measurements. Journal of Biological Systems, 15(04): 551-571.

21 Chen Y., Huang S., Pickwell-MacPherson E., 2010. Frequency-wavelet domain deconvolution for terahertz reflection imaging and spectroscopy. Optics express, 18(2): 1177-1190.

22 Chen Y., Pickwell-MacPherson E., 2009. Stationary-wavelet regularized inverse filtering: A robust deconvolution approach for terahertz reflection imaging. 34th International Conference on Infrared, Millimeter, and Terahertz Waves, 1-2. 
23 Chen Y., Sun Y., Pickwell-Macpherson E., 2010. Improving extraction of impulse response functions using stationary wavelet shrinkage in terahertz reflection imaging. Fluctuation and Noise Letters, 9(04): 387-394.

24 Zheng Y, Tay D B H, Li L. 2000. Signal extraction and power spectrum estimation using wavelet transform scale space filtering and Bayes shrinkage. Signal Processing, 80(8): 1535-1549.

25 Yang K J, Fang W P, Huang Y C, Qiao H W., 2009. A wide adaptability deconvolution technique for ultrasonic nondestructive testing. Journal of Zhejiang University (Engineer Science), 43(10): 1766-1771

26 Withayachumnankul W., Ferguson B., Rainsford T., et al., 2005. Material parameter extraction for terahertz time-domain spectroscopy using fixed-point iteration. Conference on Photonic Materials, Devices, and Applications, Proc. of SPIE, Vol. 5840: $221-231$.

28 Wallace V P, MacPherson E, Zeitler J A, et al., 2008. Three-dimensional imaging of optically opaque materials using nonionizing terahertz radiation. J. Opt. Soc. Am. A, 25(12): 3120-3133.

29 Chen Y., Sun Y., Pickwell-MacPherson E., 2011. Total variation deconvolution for terahertz pulsed imaging. Inverse Problems in Science and Engineering, 19(2): 223-232.

29 Parrott E. P. J., Sy S. M. Y., Blu T., et al., 2011. Terahertz pulsed imaging in vivo: measurements and processing methods. Journal of biomedical optics, 16(10): 106010-106010-8.

30 Pickwell-MacPherson E., 2011. Terahertz pulsed imaging in vivo. Proc. of SPIE, Vol. 7897, 78970C

31 Iwata T., Yoshioka S., Nakamura S., et al., 2013. Prediction of the thickness of a thin paint film by applying a modified partial-least-squares-1 method to data obtained in terahertz reflectometry. Journal of Infrared, Millimeter, and Terahertz Waves, 34(10): 646-659.

32 Chady T, Lopato P, Szymanik B., 2012. Terahertz and thermal testing of glass-fiber reinforced composites with impact damages. Journal of Sensors, Vol. 2012, 1-14

33 Roth D. J., Seebo J. P., Trinh L. B., et al., 2007. Signal processing approaches for Terahertz data obtained from inspection of the shuttle external tank thermal protection system foam. Review of Progress in Quantitative Nondestructive Evaluation. 
AIP Publishing, 894(1): 415-424.

34 Ren Z, Chen J, Tang X, et al., 2000. Fault feature extracting by wavelet transform for control system fault detection and diagnosis. Proceedings of the 2000 IEEE International Conference on Control Applications: 485-489.

$35 \mathrm{Lu}$ Y, Ye L, Su Z, et al., 2008. Quantitative assessment of through-thickness crack size based on Lamb wave scattering in aluminium plates. Ndt \& E International, 41(1): 59-68.

36 Kultyshev A. S., 1990. Envelope, frequency, and phase representation in terms of a family of oscillations. Measurement Techniques, 33(8): 839-842.

37 Guihong Q, Dali Z, Pingfan Y., 2001. Medical image fusion by wavelet transform modulus maxima. Optics Express, 9(4): 184-190.

38 Nason, G. P., Silverman, B. W., 1995. The stationary wavelet transform and some statistical applications. Wavelets and statistics, 281-299. Springer, New York.

39 Nema, M., Gupta, L., Trivedi, N. R., 2012. Video Compression using SPIHT and SWT Wavelet. Int. J. Electron. Comm. Eng., 5(1): 1-8.

40 Zhong, S., Oyadiji, S. O., 2007. Crack detection in simply supported beams without baseline modal parameters by stationary wavelet transform. Mech. Syst. Signal Pr., 21(4), 1853-1884.

41 Peng Z K, Chu F L, Peter W T., 2007, Singularity analysis of the vibration signals by means of wavelet modulus maximal method. Mech. Syst. Signal Pr., 21(2): 780-794.

42 Tang Y Y, Yang L, Liu J., 2000. Characterization of Dirac-structure edges with wavelet transform. Systems, Man, and Cybernetics, Part B: Cybernetics, IEEE Transactions on, 30(1): 93-109.

43 S. Mallat, W.L. Hwang, 1992. Singularity detection and processing with wavelet. IEEE Trans. Inform. Theory, 38 (2), $617-643$.

44 Korkut, E., Atlar, M., 2012. An experimental investigation of the effect of foul release coating application on performance, noise and cavitation characteristics of marine propellers, Ocean Eng., 41, 1-12. 
45 Paik, B. G., Kim, K. S., Kim, K. Y., Ahn, J. W., Kim, T. G., Kim, K. R., Jang, Y. H., Lee, S. U., 2011. Test method of cavitation erosion for marine coatings with low hardness. Ocean Eng., 38(13), 1495-1502.

46 Fan M. B., Cao B. H., Tian G. Y., 2017. Enhanced Measurement of Paper Basis Weight Using Phase Shift in Terahertz Time-Domain Spectroscopy. J. Sensors, 2017, 1-17

47 Tu, W., Zhong, S., Shen, Y., Zhou, Q., Yao, L., 2014. FDTD-based quantitative analysis of terahertz wave detection for multilayered structures. J. Opt. Soc. Am. A, 31: 2285-2293.

48 Tu, W. L., Zhong, S. C., Shen, Y. C., Incecik A., 2016. Nondestructive Testing of Marine Protective Coatings Using Terahertz Waves with Stationary Wavelet Transform, Ocean Eng., 111:582-592

49 Momose, W., Yoshino, H., Katakawa, Y., et al., 2012. Applying terahertz technology for nondestructive detection of crack initiation in a film-coated layer on a swelling tablet. Results in Pharma Sciences, 2: 29-37.

50 Tsai, T. R., Chen, S. J., Chang, C. F., Hsu, S. H., Lin, T. Y., Chi, C. C., 2006. Terahertz response of GaN thin films. Opt. Express, 14(11): 4898-4907.

51 Karpowicz N, Zhong H, Xu J, et al., 2005. Comparison between pulsed terahertz time-domain imaging and continuous wave terahertz imaging. Semiconductor Science and Technology, 20(7): S293. 